Journal of Applied Pharmaceutical Science Vol. 6 (07), pp. 171-177, July, 2016

Available online at http://www.japsonline.com

DOI: $10.7324 / \mathrm{JAPS} .2016 .60725$

ISSN 2231-3354 (cc) BY-NC-SA

\title{
Fabrication and Characterization of chitosan based polymeric Escitalopram nanoparticles
}

\author{
Rashi Rajput, Sachin Kumar, Payal Nag, Manisha Singh* \\ Department of Biotechnology, Jaypee Institute of Information Technology, Noida - 201307, India.
}

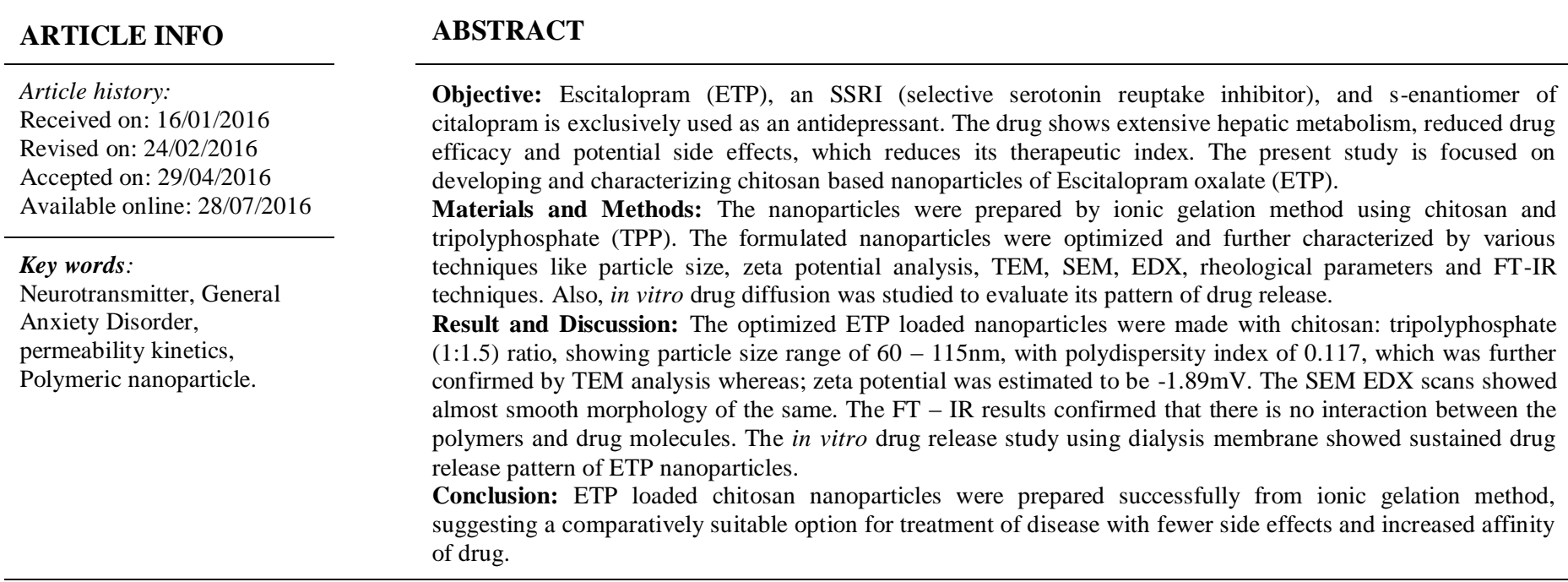

\section{INTRODUCTION}

Depression and anxiety have been incorporated in our daily lifestyle now and are faced by people of all age groups, similarly it affects both men and women across the world, but various research studies showed higher occurrences in women than in men (Taylor et al., 2008). It has various forms like general anxiety disorder (GAD), obsessive compulsive disorder (OCD), major depressive disorder (MDD), social anxiety disorder (SAD) etc. which prevails in $\sim 5-7 \%$ of world population. Selective serotonin reuptake inhibitor (SSRI) class of

* Corresponding Author

Manisha Singh, Assistant Professor, Department of Biotechnology, Jaypee Institute of Information Technology, Noida - 201307, India Email:manishasingh1295@gmail.com drugs like Citalopram, Escitalopram, Paroxetine, Fluoxetine, Fluvoxamine, Sertraline etc are widely used to treat these anxiety and depressive disorders. Among these classes of drugs Escitalopram oxalate (ETP) is the most used form of SSRI category of drugs. It has been approved by FDA as an antidepressant drug and is used in treatment of general anxiety disorder (GAD), major depressive disorder (MDD).

Escitalopram is an S- enantiomer (single isomer) of the racemic bicyclic phthalane derivative citalopram but it is highly metabolised in liver by enzymes like CYP2C19, CYP3A4 and CYP2D6 and forms metabolites like S-demethylcitalopram (SDCT) and S-didemethylcitalopram (S-DDCT). It is said to be 7 and 27 times more effective than S-DCT and S-DDCT, respectively, in inhibiting the serotonin reuptake (Burke et al., 2002; Montgomery et al., 2008). 
Moreover, it has a bioavailability of $80 \%$ with plasma protein binding of $56 \%$ and is eliminated through renal path. But there arises the need for targeted nanosized formulation to further secure its therapeutic index and prevent it from chemical and enzymatic degradation.

Also, it has been reported that drug delivery through nanoparticles are more effective as it can easily cross the biological barriers as in case of lansoprazole nanoparticles whose pharmaceutical efficiency increased by encapsulating it in polymeric nanoparticles (Nagarajan et al., 2015). The benefits of using the polymeric carriers are that they are biocompatible, biodegradable, non-immunogenic, non-toxic and water soluble (Agnihotri et al., 2004). Thus, chitosan polymer remains an obvious choice for the same and aim of present study was to prepare chitosan nanoparticles by ionic gelation method for enhanced bioavailability and lesser side effects with lower hepatic metabolism (Llorca et al., 2005).

\section{MATERIALS AND METHODS}

Chitosan, Tri Polyphosphate (TPP), Monosodium phosphate anhydrous and Disodium phosphate anhydrous were purchased from Himedia Laboratories, Mumbai, India. Dialysis Membrane (9777) was from Sigma Aldrich, USA. Escitalopram oxalate (ETP) was obtained as gift from Jubilant Life Sciences, India. Acetic acid, Methanol and all other chemical used were of analytical grade.

\section{Preparation of ETP loaded chitosan nanoparticles}

ETP loaded chitosan nanoparticles were prepared by ionic gelation method using chitosan and TPP polymers for cross linkages. The crosslinkage lattice is formed between two ionic polymers of opposite charge, (chitosan as cationic and TPP as anionic) bound to each other by ionic bonds (Baby et al., 2012). Chitosan gel solution was prepared by dissolving chitosan(1$2.5 \mathrm{mg} / \mathrm{ml})$ in $5 \%(\mathrm{v} / \mathrm{v})$ glacial acetic acid and stirred continuously at $600 \mathrm{rpm}$ overnight to get a clear chitosan gel solution.

ETP drug $(1.5-5 \mathrm{mg} / \mathrm{ml})$ was then added in chitosan solution and further stirred for 40 minutes. After this, sodium tripolyphosphate (TPP) solution $(0.5-2.5 \mathrm{mg} / \mathrm{ml})$ was prepared in distilled water and added drop wise with constant speed of $0.8 \mathrm{ml} / \mathrm{minute}$ to the chitosan solution under constant stirring for 40 minutes again. Further it was sonicated at frequency of $10,000 \mathrm{MHz}$ for 15 minutes. The prepared solution was centrifuged at $12750 \mathrm{~g}$ for 40 minutes and the supernatant was discarded, this washing step was repeated thrice and then finally pellets were lyophilised for further characterization (Katas et al., 2013).

\section{Characterization of optimised nanoparticles Drug encapsulation efficiency}

The drug encapsulation efficiency (EE) of the prepared nanoparticles was determined by centrifuging the nanoparticles at $12750 \mathrm{~g}$ for $40 \mathrm{~min}$ and analysing the supernatant at 238nm, (Katas et al., 2013). Then encapsulation efficiency (EE) was calculated using the following equation:

Encapsulation efficiency $(\%)=$

Total amount of drug loaded - free drug in supernatant $\times 100$

Total amount of drug loaded

\section{Particle size and zeta potential analysis}

The particle size analysis was performed using dynamic light scattering (DLS) technique, which is based on brownian motion of molecules, dispersed in liquid and relates this to the size of the particles by illuminating the particles with a laser light and analyzing the intensity fluctuations in the scattered light (Gevariya et al., 2011). The particle size along with zeta potential analysis of optimised nanoparticles was done on Malvern Zetasizer (Nano ZS). Zeta potential (ZP) shows the electro phoretic particle velocity in an electrical field where the particle obtains a charge due to the dissipation of the counter ions on the surface of molecule (Saha et al., 2010).

\section{Transmission electron microscopy (TEM)}

Transmission electron microscopy (Morgagni 268D) analysis was done to find out the morphology of the nanoparticles and also to confirm the size range of the drug loaded nanoparticles. The optimised ETP loaded nanoparticle was further diluted (1: 50) by distilled water and ultrasonicated for 15 minutes. It was then stained with $2 \%$ phosphotungstic acid and a drop of sample was then fixed on 300 mesh carbon-coated copper grid. The images of representative areas were taken at suitable magnifications $(200 \mathrm{~nm})$ (Saha et al., 2010)

\section{Scanning electron microscopy (SEM) and energy-dispersive spectroscopy $(\mathrm{EDX})$}

Scanning electron microscopy (ZEISS EVO 40) was done to study the topographical and compositional arrangements whereas, EDX (PANalytical epsilon 5) scan was performed to have elemental analysis of optimised nanoparticles. A drop of the nanoparticles suspension was placed on a metallic surface followed by air drying under vacuum, coated with gold sputtering (Verma and Ram, 2010).

\section{Fourier transform infrared spectroscopy (FTIR)}

Fourier transform infrared spectroscopy (IR-810, JASCO, Tokyo in SAIF, Panjab University) spectra of ETP drug, chitosan nanoparticles without drug and optimised ETP loaded chitosan nanoparticles were scanned. The samples were prepared by potassium bromide disc method and scanned for absorbance from the range of $400-4000 \mathrm{~cm}^{-1}$ (Gevariya et al., 2011).

\section{Rheological parameters}

Different physico-chemical parameters like $\mathrm{pH}$, conductivity and density of optimised nanoparticle were measured. $\mathrm{pH}$ and conductivity of the samples were measured using $\mathrm{pH}$ meter (Thermo Orion 420A+) whereas, density was measured using specific density bottle (Borosil). The viscosity was measured with a 
Brookfield rotational viscometer (LVDV, Brookfield Inc., USA). The measurement was done at $30^{\circ} \mathrm{C}$ at $5 \mathrm{rpm}$ viscosity (Lee et al., 2004 \& Li and Huang, 2011).

\section{In vitro drug release studies}

In vitro drug absorption and permeability analysis was performed by using Franz diffusion cell with pre treated dialysis membrane (Sigma9777). This was mounted between donor and receiver compartments held together on both sides with a clamp. The receiver compartment was completely filled with PBS buffer (pH 7.2) and kept under continuous stirring whereas, ETP loaded nanoparticle solution was filled in donor compartment to get diffused through the semi permeable dialysis membrane.

Diffused nanoparticle samples $(1 \mathrm{ml})$ were collected at pre determined time intervals $(0-24$ hours $)$ from the sampling port and reloaded it with equal volume of PBS $(1 \mathrm{ml})$ again (Verma and Ram,2010). The collected samples were then analysed at $238 \mathrm{~nm}$.

\section{RESULT AND DISCUSSION}

\section{Optimization of process parameters}

After optimization of process parameters (Table1), most appropriate formulation A3 was selected as it showed the highest encapsulation efficiency of $\sim 79 \%$ with polymer concentration of 1:1.5 (ratios of chitosan and TPP concentration) (Table2) and $2.5 \mathrm{mg} / \mathrm{ml}$ of drug concentration.

Table 1: Volume optimization of polymeric solutions

\begin{tabular}{cccccc}
\hline \multirow{2}{*}{ Ratios } & \multicolumn{5}{c}{ \% Encapsulation efficiency } \\
\cline { 2 - 6 } & A1 & A2 & A3 & A4 & A5 \\
\hline $1: 1$ & 13.4 & 16.4 & 34.7 & 53.2 & 42.57 \\
$1: 1.5$ & 24.7 & 47.2 & 49.42 & 49.42 & 52.7 \\
$1: 2$ & 31.2 & 45.4 & 48.8 & 53.4 & 57.4 \\
$1: 2.5$ & 50.86 & 52.71 & 79.14 & 60 & 60.02 \\
$1: 3$ & 28.6 & 35.4 & 52.14 & 43.8 & 48.8 \\
\hline
\end{tabular}

It was concluded from the optimization data that as the amount of Chitosan and TPP concentration increased the encapsulation efficiency (EE) decreased proportionally (Figure1) which could be due to the reason that increased concentrations of polymers would have inhibited the stable covalent ionic bond formation whereas, in optimised ratio $(1: 1.5)$ there is a prevalence of higher degree of encapsulation efficiency due to the availability of one chitosan molecule with two molecules of tripolyphosphate to form a cross linking structure of nanoparticles. Similarly, in case of drug concentration the maximum EE was observed at dose of $2.5 \mathrm{mg} / \mathrm{ml}$. (Shu and Zhu, 2000).

Table 2: Optimization of process parameters (Polymer and drug concentration) with respect to encapsulation efficiency.

\begin{tabular}{|c|c|c|c|c|}
\hline \multirow{2}{*}{ Composition } & \multicolumn{4}{|c|}{ Entrapment efficiency (\%) with Drug Concentration } \\
\hline & $1.5 \mathrm{mg} / \mathrm{ml}$ & $2.5 \mathrm{mg} / \mathrm{ml}$ & $3.5 \mathrm{mg} / \mathrm{ml}$ & $5 \mathrm{mg} / \mathrm{ml}$ \\
\hline \multicolumn{5}{|c|}{ Chitosan $(1 \mathrm{mg} / \mathrm{ml})$ and varied TPP concentration } \\
\hline A1 & 61.2 & 50.86 & 48.3 & 45.7 \\
\hline $\mathrm{A} 2$ & 65.7 & 52.708 & 49.2 & 46.1 \\
\hline A3 & 82.5 & 79.14 & 61.9 & 54.5 \\
\hline A4 & 69 & 60 & 46.9 & 57 \\
\hline A5 & 51.6 & 42.568 & 40.1 & 38.6 \\
\hline \multicolumn{5}{|c|}{ Chitosan $(1.5 \mathrm{mg} / \mathrm{ml})$ and varied TPP concentration } \\
\hline B1 & 46.8 & 23.428 & 41.6 & 41.7 \\
\hline B2 & 45.4 & 33.428 & 39.4 & 38.4 \\
\hline B3 & 43.3 & 34.288 & 43.3 & 42.8 \\
\hline B4 & 39.4 & 30.568 & 41.3 & 31.6 \\
\hline B5 & 35.1 & 34.288 & 32.9 & 31.7 \\
\hline \multicolumn{5}{|c|}{ Chitosan $(2 \mathrm{mg} / \mathrm{ml})$ and varied TPP concentration } \\
\hline $\mathrm{C} 1$ & 28.7 & 19.428 & 31.2 & 25.1 \\
\hline $\mathrm{C} 2$ & 23.1 & 16.428 & 30.7 & 20.8 \\
\hline $\mathrm{C} 3$ & 25.8 & 29.428 & 36.38 & 21.6 \\
\hline $\mathrm{C} 4$ & 25 & 24.428 & 26.1 & 24.2 \\
\hline C5 & 23.6 & 20.86 & 24.1 & 19.8 \\
\hline \multicolumn{5}{|c|}{ Chitosan $(2.5 \mathrm{mg} / \mathrm{ml})$ and varied TPP concentration } \\
\hline D1 & 25.4 & 22.708 & 18.2 & 15.9 \\
\hline D2 & 21.3 & 27.708 & 14.6 & 18.2 \\
\hline D3 & 31 & 30 & 24.1 & 28 \\
\hline D4 & 30.6 & 30.288 & 6.3 & 26 \\
\hline D5 & 24.9 & 30.14 & 10.3 & 19.1 \\
\hline
\end{tabular}

\section{Characterization of nanoparticles}

Particle size (PS), Poly dispersibility index (PDI) and Zeta potential (ZP) analysis Average particle size (PS) of optimised ETP loaded chitosan nanoparticles (A3) was $93.63 \pm$ $1.04 \mathrm{~nm}$ which was further confirmed by TEM. The PDI score of the same was recorded as $0.117 \pm 0.06$ showing good homogeneity and dispersibility of nanoparticles in the solution (Figure3a) (Gevariya et al., 2011). Whereas, Zeta potential of A3 formulation was $-1.89 \pm 0.27 \mathrm{mV}$ (Figure $3 \mathrm{~b}$ ) indicating the surface electrical charge (negative) due to ionization or dissociation of surface groups (carboxyl and/or amino and phosphate groups) along with total molecular charge (positive or negative).Therefore, from the recorded observations, it can be suggested that the optimised formulation (A3) is highly stable with less ionic charge. Also, many earlier research studies had reported that there is nonaggregation of nanoparticles in the range of $\pm 30 \mathrm{mV}$ (Saha et al., 2010).

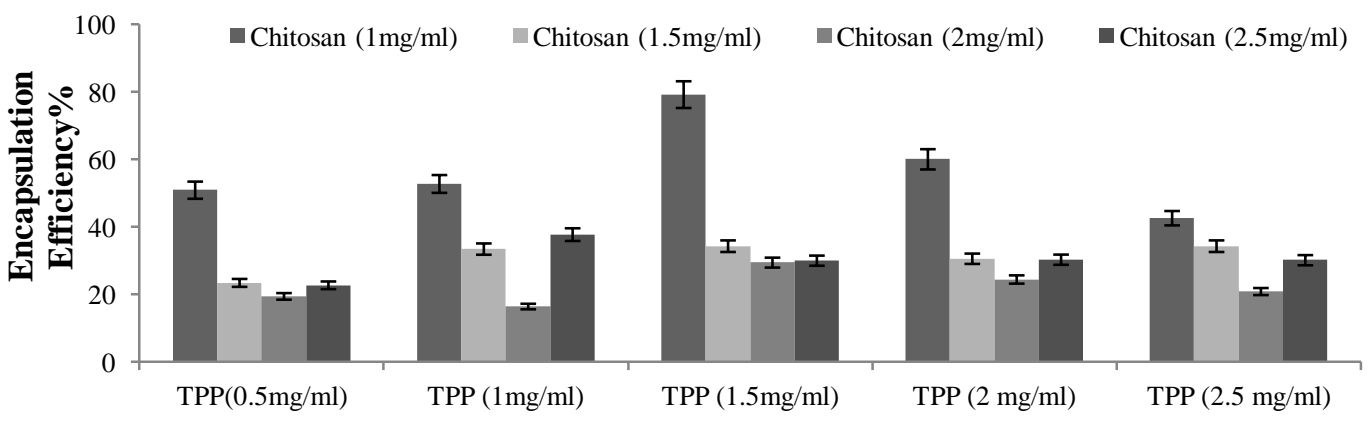

Concentration of TPP

Fig. 1: Comparison between different chitosan-TPP formulations based on encapsulation efficiency. 


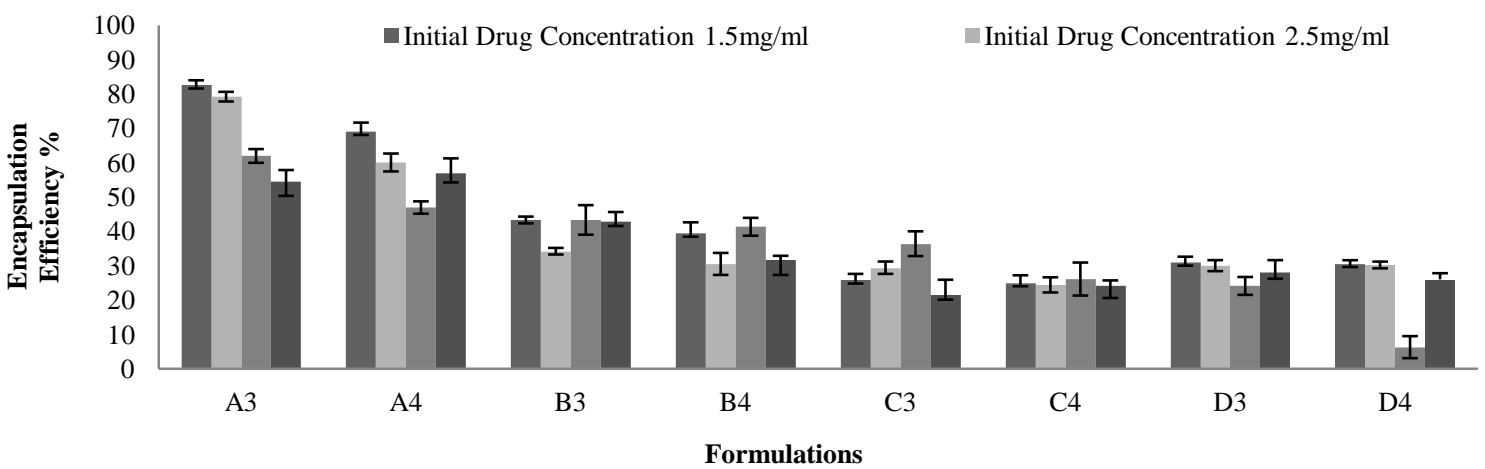

Fig. 2: Comparison between different drug concentrations based on encapsulation efficiency.

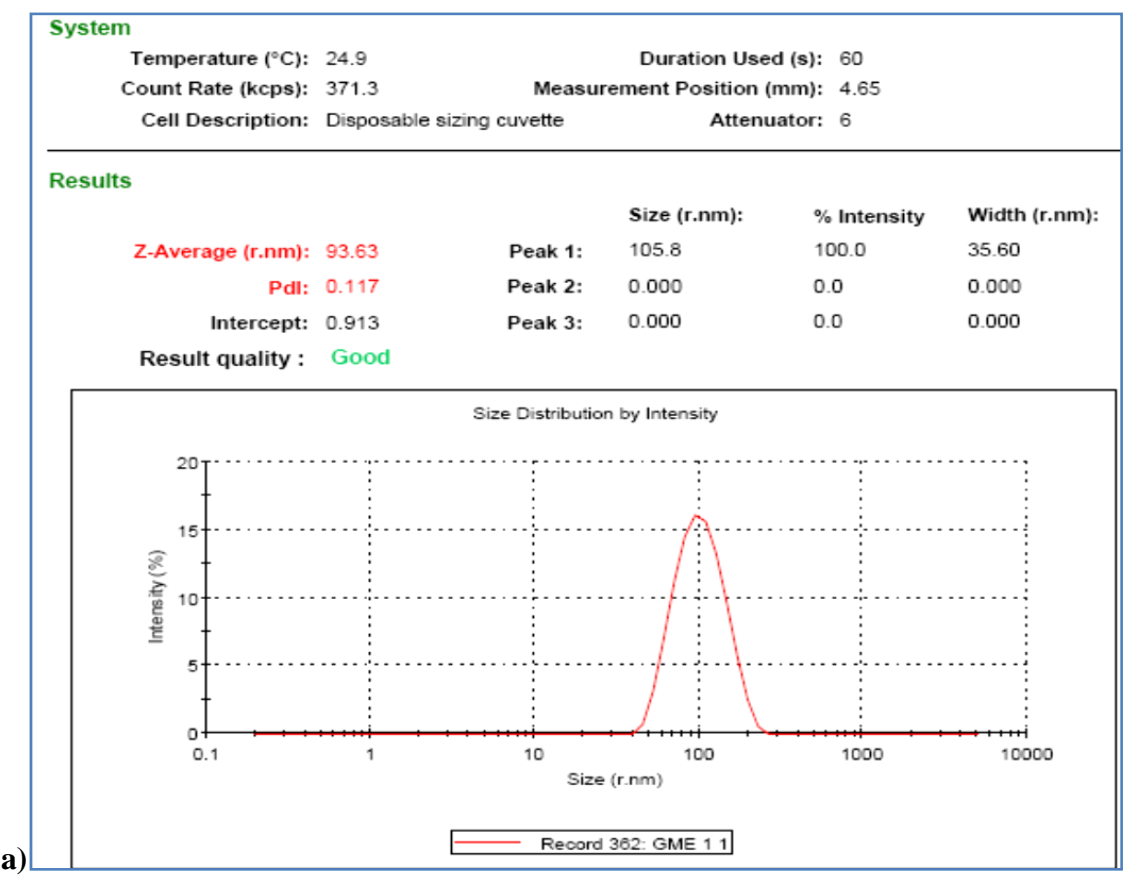

(a)

\begin{tabular}{|c|c|c|c|c|}
\hline \multicolumn{5}{|l|}{ Results } \\
\hline & & Mean $(\mathrm{mV})$ & Area $(\%)$ & Width (mV) \\
\hline Zeta Potential $(\mathrm{mV}):-1.89$ & Peak 1: & -1.89 & 100.0 & 3.55 \\
\hline Zeta Deviation $(\mathrm{mV}): 3.55$ & Peak 2: & 0.00 & 0.0 & 0.00 \\
\hline Conductivity $(\mathrm{mS} / \mathrm{cm}): 0.174$ & Peak 3: & 0.00 & 0.0 & 0.00 \\
\hline
\end{tabular}

Result quality : Good

Zeta Potential Distribution

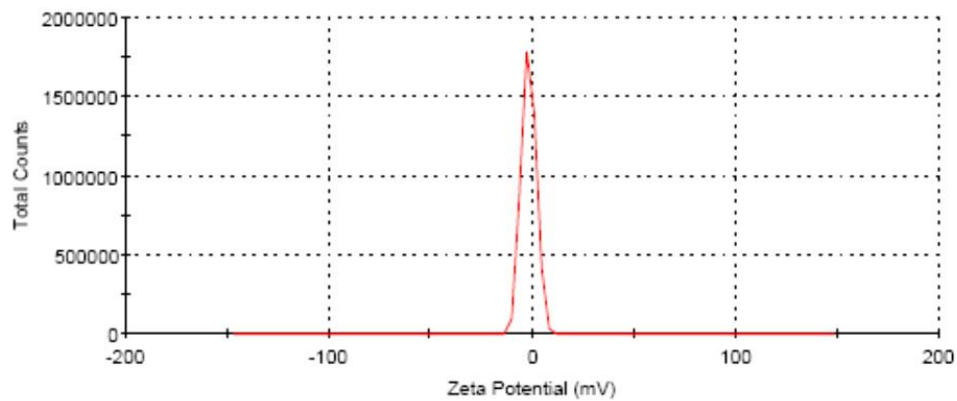

(b)

Fig. 3: (a)-Particle Size of optimized nanoparticles (A3) was $93.63 \eta \mathrm{m}$ and and (b)-zeta potential of optimized nanoparticles (A3) was $-1.89 \mathrm{mV}$ with PDI 0.117 and conductivity $0.174 \mathrm{mS} / \mathrm{cm}$. 


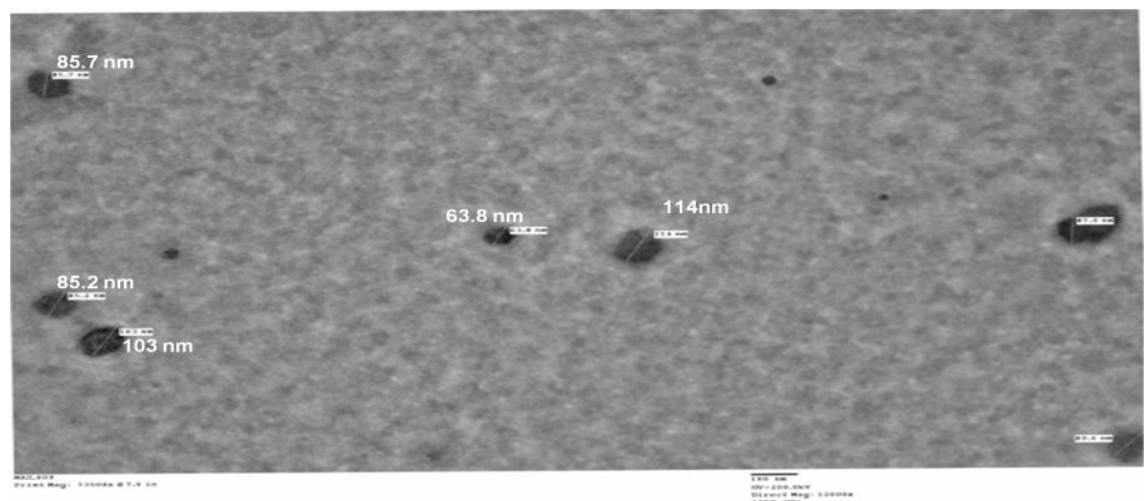

Fig. 4: Transmission electron spectroscopy (TEM) analysis done for A3 formulation.
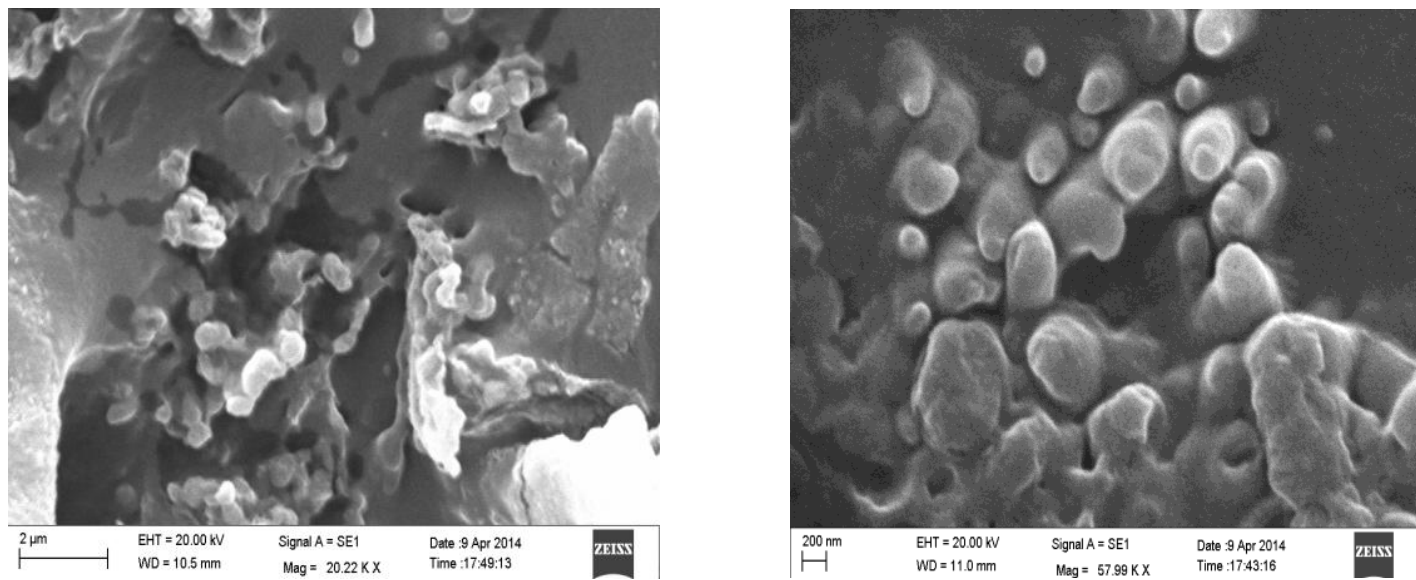

Fig. 5: (a)-Base image of SEM analysis and (b)-Magnified image of SEM analysis
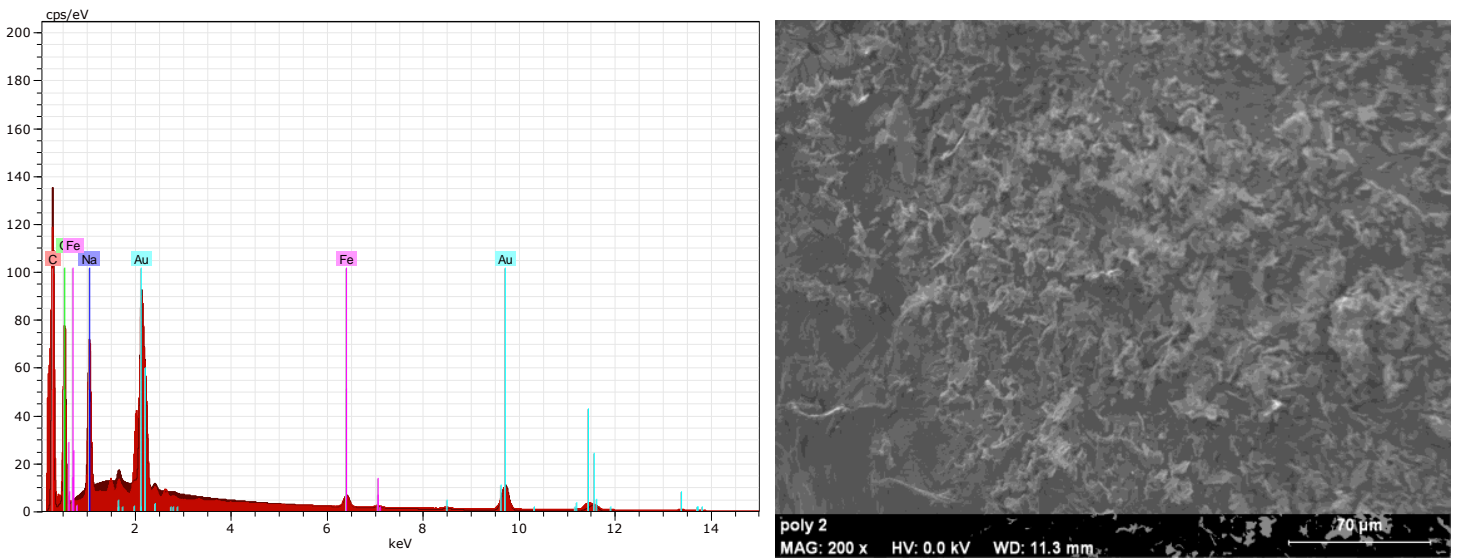

Fig. 6: EDX analysis showing localized chemical composition and topological image of nanoparticles.

\section{Transmission electron microscopy (TEM)}

The TEM micrograph obtained from the imaging showed that the droplet size of the samples were in nanometric range (60 $115 \mathrm{~nm}$ in diameter) (Figure 4).

These results were in accordance with the DLS findings, which recorded the average mean of PS as $93.63 \pm 1.04 \mathrm{~nm}$. The nanoparticle image appeared with almost spherical morphology of droplets, hence acquiring minimum surface area and indicating the strong possibility of permeating through any biological barriers (Saha et al., 2010).

\section{Scanning electron microscopy (SEM) and energy-dispersive spectroscopy $(\mathrm{EDX})$}

SEM technique is used for the morphological characterization of particles, thereby using a high energy electron beam to scan over the surface (Verma and Ram, 2010). The results obtained from SEM indicates the almost spherical and smooth morphology of nanoparticles when observed in the scale of 200 $\mathrm{nm}$ (Figure 5a) although it appears to be irregular when observed in the scale of $2 \mu \mathrm{m}$ so it can be concluded that morphologically the particles are almost smooth and spherical (Figure 5b). 


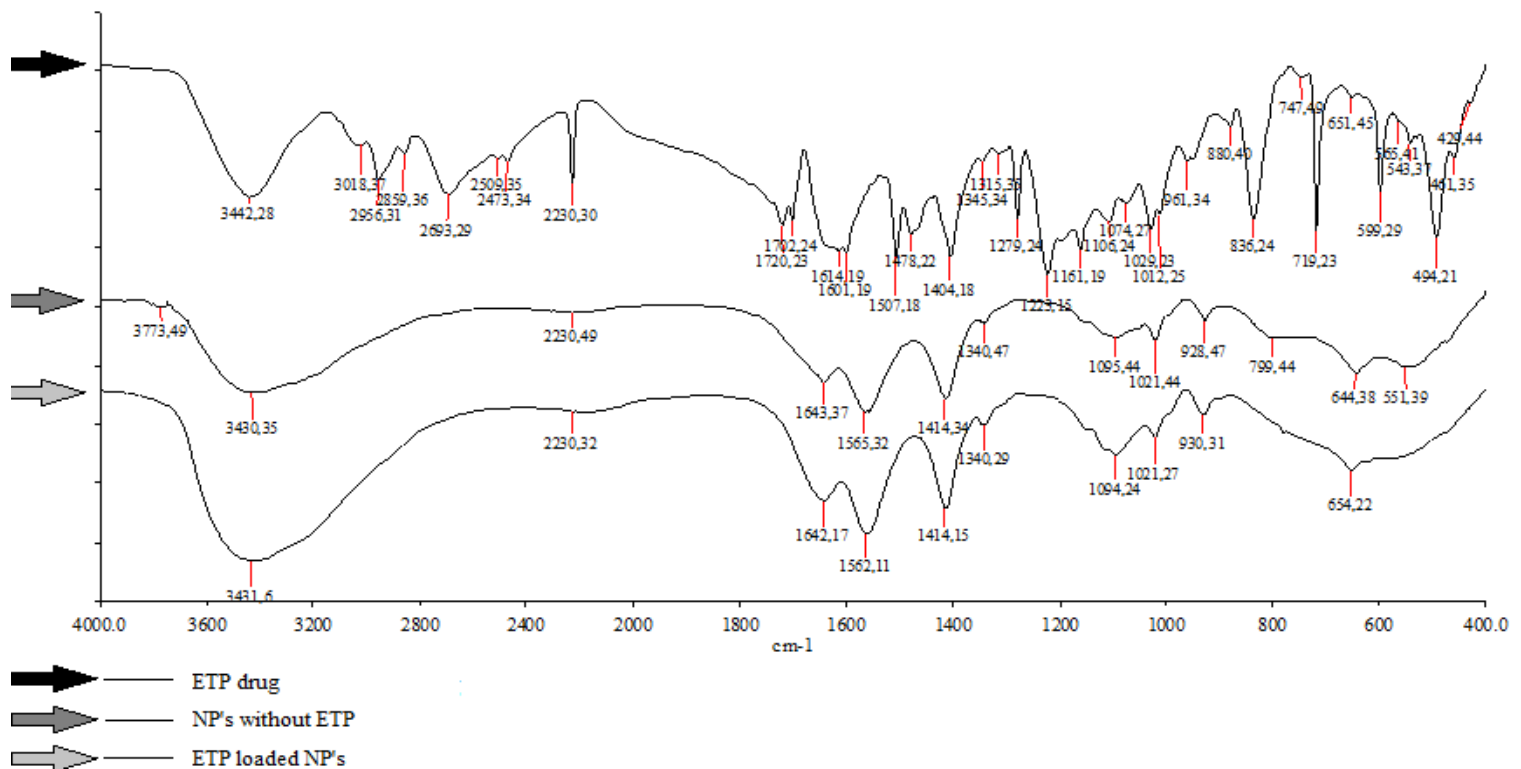

Fig. 7: FT-IR spectra analysis for ETP loaded chitosan nanoparticles.

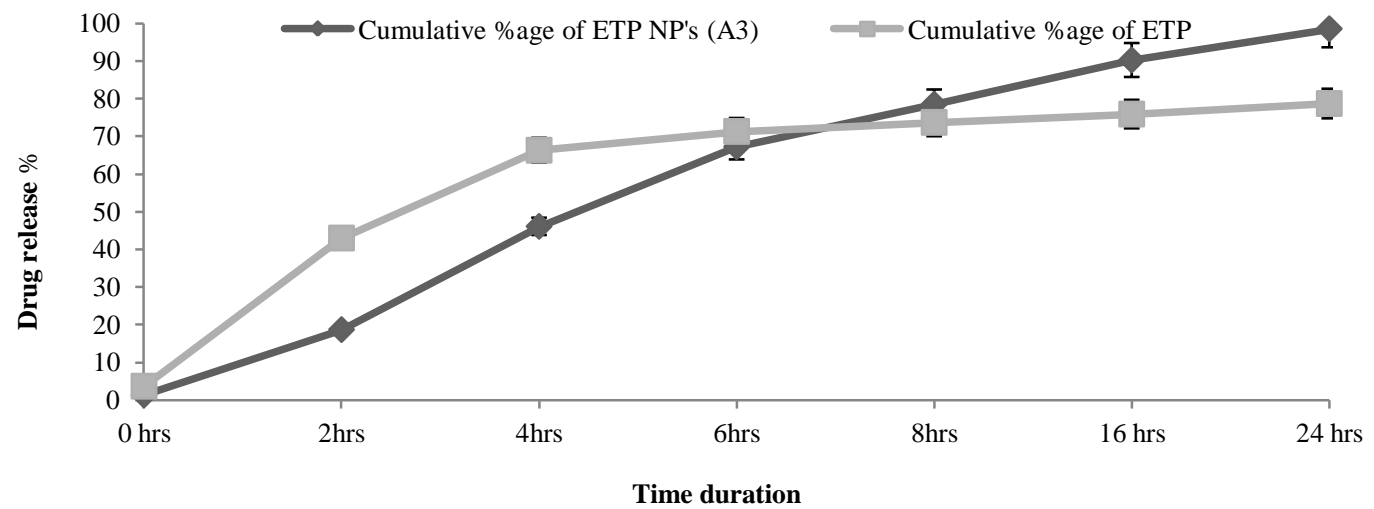

Fig. 8: Graph depicting release kinetics of drug in nanoparticle formulations as compared to normal drug.

Whereas, in EDX spectrometry scanning prominent peaks of $\mathrm{C}$ and $\mathrm{Na}$ indicates the presence of cross linkage between the chitosan and TPP and confirms the formation of chitosan nanoparticles whereas, Au appearance is due to gold sputtering done (Figure 6).

\section{Fourier transform infrared spectroscopy (FTIR)}

The graphical representation of FT-IR spectra of ETP drug, NP's without drug and ETP loaded NP's (A3) attributed to the linkage between phosphoric and ammonium ions concluding that the di polyphosphate groups of TPP are linked with ammonium groups of chitosan. Moreover it also showed non emergence of signature peaks of drug 3431, 2230 and 1414 representing $\mathrm{OH}$ ( $\mathrm{H}$ bond), $-\mathrm{C} \equiv \mathrm{C}-$, and $-\mathrm{C}-\mathrm{C}$ - stretch in ring (aromatic) groups in optimised ETP loaded NP's. In addition to the same, emergences of all the prominent peaks in ETP loaded NP's were quite similar with nanoparticles without drug, suggesting less possibility of ETP available on surface of NP's. (Figure 7) (Gevariya et al., 2011).

\section{Rheological parameters}

Analysis of physicochemical properties is important for efficient drug release from nanoparticles through various biological and physical models (Table3).

Table 3: Physicochemical parameters of nanoparticles.

\begin{tabular}{lcccc}
\hline \multicolumn{1}{c}{ Sample Code } & $\mathbf{p H}$ & $\begin{array}{c}\text { Density } \\
(\mathbf{g} / \mathbf{m l})\end{array}$ & $\begin{array}{c}\text { Conductivity } \\
(\boldsymbol{\mu} \mathbf{S} / \mathbf{c m})\end{array}$ & Viscosity(cP) \\
\hline $\begin{array}{l}\mathrm{C}-1 \mathrm{mg} / \mathrm{ml}, \\
\mathrm{TPP}-1.5 \mathrm{mg} / \mathrm{ml}(\mathrm{A} 3)\end{array}$ & 6.7 & 0.976 & 174.8 & 0.887 \\
\hline
\end{tabular}

Therefore, parameters like $\mathrm{pH}$, density, conductivity and viscosity were measured. The $\mathrm{pH}$ of the formulation (A3) was recorded as 6.7 showing proximity with neutral (pH-7.3) (Katas et al., 2013 \& Fan et al., 2012), similarly conductivity of the same was $0.174 \mathrm{mS} / \mathrm{cm}$ which is quite less than the conductivity of blood (blood plasma conductivity $12 \mathrm{mS} / \mathrm{cm}$ ) (Jaffrin and Fournier, 1999), hence allowing the nanoparticles to easily flow through blood vessels without any repulsion. The density observed was $0.976 \mathrm{~g} / \mathrm{ml}$, equivalent to that of water $(1 \mathrm{~g} / \mathrm{ml})$ and is suitable for 
administration through any of the delivery route whereas, the obtained viscosity of the sample $(0.887 \mathrm{cP})$ is reported to show good flowability and can easily pass through any biological barrier (Lee et al., 2004; Li and Huang, 2011).

\section{In vitro drug release studies}

In vitro drug release pattern of Escitalopram (ETP) and ETP loaded was studied to check permeability of pure drug (ETP) and its optimized nanoparticles (A3) through dialysis membrane, it showed $78.6 \pm 2.6 \%$ release (24 hours) of drug in receptor compartment whereas, for ETP loaded nanoparticles it was $98.4 \pm$ $1.07 \%$ release in 24 hours (Figure8), proposing a typical linear diffusion profile of nanoparticles through the dialysis membrane (Verma and Ram, 2010). The expected characteristic of nanoparticles of sustained release was verified. However, diffusion equilibrium was attained after 6 hours with ETP drug, compared to nanoparticles (A3). Results further propose sustained release of drug molecules using nanoparticle systems.

\section{CONCLUSION}

In this study escitalopram nanoparticles were prepared to decrease the loss of drug entering in systemic circulation due to high hepatic metabolism of the drug by getting entrapped in nanoparticles, becoming more stable and shielded from enzymatic degradation. The optimized nanoparticle formulation of chitosan and tri polyphosphate $(1: 1.5)$ with $2.5 \mathrm{mg}$ drug (ETP) showed particle size in nanometric range $(60-115 \mathrm{~nm})$ and exhibited approximately $98.4 \pm 1.07 \%$ of drug release from nanoparticles in 24 hours from the dialysis membrane. Also physicochemical study revealed that $\mathrm{pH}(6.7)$, conductivity $(0.174 \mathrm{mS} / \mathrm{cm})$, density $(0.976 \mathrm{~g} / \mathrm{ml})$ and viscosity $(0.887 \mathrm{cP})$ of optimised nanoparticles (A3) was equally suitable for all the route of drug administration including oral, systemic and intranasal drug delivery. Further FTIR and SEM analysis showed no bond formation between the drug molecules and polymers on the surface and almost smooth morphology of the nanoparticles respectively. Therefore, these nanoparticles being in nanometric size range can penetrate through various biological barriers and could serve as a potential delivery system for the treatment of anxiety disorders.

\section{ACKNOWLEDGMENTS}

The research group is grateful to the Department of Biotechnology and Bioinformatics of Jaypee Institute of Information Technology, Noida, Uttar Pradesh for providing necessary facilities to execute this work.

\section{REFERENCES}

Agnihotri SA, Mallikarjuna NN, Aminabhavi TM. Recent advances on chitosan-based micro-and nanoparticles in drug delivery. J Control Release, 2004;100:5-28.

Baby B, Harsha NS, Jayaveera KN, Abraham A. Formulation and Evaluation of Levofloxacin Nanoparticles by Ionic Gelation Method. J. Pharmacy and Pharmaceutical Sciences, 2012; 1(1).

Burke WJ, Kratochvil CJ. Stereoisomers in Psychiatry: The Case of Escitalopram. Prim Care Companion J Clin Psychiatry, 2002; 4: 20-24.

Fan W, Yan W, Xu Z, Ni H. Formation mechanism of monodisperse, low molecular weight chitosan nanoparticles by ionic gelation technique. Colloids and Surfaces B: Biointerfaces,2012;90:21-27.

Gevariya HB, Gami S, Patel N. Formulation and characterization of levofloxacin-loaded biodegradable nanoparticles. Asian J Pharm. 2011; 2: 114-119.

Jaffrin MY, Fournier C. Comparison of optical, electrical, and centrifugation techniques for haematocrit monitoring of dialysed patients Med. Biol. Eng. Comput., 1999, 37: 433-439.

Katas H, Raja M A G, Lam KL. Development of Chitosan Nanoparticles as a Stable Drug Delivery System for Protein/siRNA. Int. J. Biomaterials, 2013.

Lee DW, Powers K, Baney R. Physicochemical properties and blood compatibility of acylated chitosan nanoparticles. Carbohydrate Polymers, 2004; 58(4): 371-377.

Li J, Huang Q. Rheological properties of chitosantripolyphosphate complexes: From suspensions to microgels. Carbohydrate Polymers, 2011; 87(2): 1670-1677.

Llorca PM, Brousse G, Schwan R. Escitalopram for treatment of major depressive disorder in adults. L.Encephale, 2005; 31:490-501.

Montgomery SA, Loft H, Sánchez C, Reines EH, Papp M. Escitalopram (S-Enantiomer of Citalopram): Clinical Efficacy and Onset of Action Predicted from a Rat Model. Pharmacology and toxicology, 2008; 88:282-286.

Nagarajan E, Shanmugasundaram P, Ravichandiran. V, Vijayalakshmi A, Masilamani K. Development and Evaluation of Chitosan Based Polymeric Nanoparticles of an Antiulcer Drug Lansoprazole. J App Pharm Sci, 2015; 5 (04): 020-025.

Saha P, Goyal AK, Rath G. Formulation and Evaluation of Chitosan- Based Ampicillin Trihydrate Nanoparticles. Tropical J. Pharmaceutical Research, 2010; 9: 483-488.

Shu X.Z, Zhu K.J. A novel approach to prepare tripolyphosphate/ chitosan complex beads for controlled release drug delivery. Int. J. Pharm. 2000; 201: 51-58.

Taylor CT, Pollack MH, LeBeau RT, Simon NM. Anxiety disorders: panic, social anxiety, and generalized anxiety. Elsevier Mosby, 2008; 1(32).

Verma VK, Ram A. Preparation, Characterization and in-vitro release of Piroxicam-loaded Solid Lipid Nanoparticles. Int. J. Pharmaceutical Sciences and Nanotechnology, 2010; 3.

\section{How to cite this article:}

Rajput R, Kumar S, Nag P, Singh M. Fabrication and Characterization of chitosan based polymeric Escitalopram nanoparticles. J App Pharm Sci, 2016; 6 (07): 171-177. 